\title{
Herbal remedies are the main etiologic factor in melanosis coli, a case series study
}

Research Article

Taylan Kav", Yusuf Bayraktar

Hacettepe University Faculty of Medicine, Department of Gastroenterology,

06100 Ankara, Turkey

Received 20 March 2009; Accepted 17 November 2009

Abstract: Melanosis coli is a brown to black discoloration of the colon mucosa usually associated with long-term ingestion of laxatives. However, melanosis coli can be found in patients with no history of laxative use. Regular use of herbal remedies could be the major source of anthranoid laxatives in such patients. We designed a prospective case series study to identify the clinical characteristics and etiology of melanosis coli in affected patients. This study took place in Ankara, Turkey, between 08/2005 and 11/2006. Patients with endoscopic diagnosis of melanosis coli were interviewed for demographical data and use of herbal remedies. A total of 380 colonoscopies were performed during this period. Melanosis coli was diagnosed endoscopically in 12 patients (3.17\%), 11 of whom were found to have characteristic pigment-laden macrophages in histopathological examination. Herbal remedies were the main etiological factor in the development of melanosis coli in 10 out of 11 patients. Diffuse involvement was found in 2 patients who had a history of long-term use. In 8 patients, melanosis coli was located in the left side of the colon. Although melanosis coli is a harmless discoloration of colonic mucosa resulting from complementary or alternative medicine, we believe that this association with herbals was overlooked or not inquired in patients. Therefore, it should be emphasized that "natural" or "alternative" is not equal to "safe".

Keywords: Melanosis coli • Herbal remedies • Laxatives • Sennosides • Etiology

(C) Versita Sp. z 0.0 .

\section{Introduction}

Colonoscopy has increasingly been used in the investigation of patients with lower gastrointestinal (GI) system symptoms like chronic constipation and diarrhea. This procedure has the advantage of obtaining biopsies for pathologic study for confirming diagnoses. Routine colonoscopy examinations may sometimes reveal Melanosis Coli (MC). MC is a well-known condition that is associated with long-term use of anthranoid-containing laxatives [1]. MC refers to brown-to-black pigmentation of colonic mucosa that can occur within several months of regular use and can last for nearly one year after discontinuation of the laxative use. This pigment is related to lipofuscin, a wear-and-tear product, which is released during cellular apoptosis and is contained in mucosal macrophages [2,3].

Because of the well-known association with anthranoid laxatives, almost all MC patients are asked about laxative abuse. MC has also been found in patients who have no history of laxative use.
The use of complementary and alternative medicine (CAM) by the general public is prevalent, and the number of patients looking for nonconventional practices to manage their chronic diseases is increasing. Among the U.S. general population, for instance, the 1-year prevalence of CAM use rose from $33 \%$ in 1990 to $42 \%$ in 1997. Therefore, it is not surprising to encounter dissatisfied patients, for whom conventional therapies have failed, searching for other possibilities [4-7].

It has been estimated that $28.9 \%$ of U.S. adults use CAM in some form. Herbal medicine is one of the most common CAM modalities. It has been estimated that between $9.6 \%$ and $12.1 \%$ of U.S. adults use some form of herbal product [5].

Herbal remedies are mostly used for gastrointestinal diseases, and these preparations often contain laxatives. There is a trend towards using a mixture of herbal teas to lose weight. A study from Turkey has found that all of the 13 commercial and handmade mixtures of dietform teas sold by herbalists contain senna in significant amounts [8]. A couple of cups of tea can contain a 
therapeutic dose of laxatives. As most patients do not inform their doctors about taking CAM, it is not surprising that information about MC is overlooked. Some of the patients with $\mathrm{MC}$ report that they do not take laxatives; however, in the case of herbal therapy, its role in this group of patients is unclear. A study from Korea aimed to determine the relation between colonic transit time and constipation and to identify the clinical characteristics of patients with MC. This study reveals anthranoid laxatives as a major cause; however, teas, herbs, and health foods comprise $30 \%$ of the cases as a cause. There is no information about the type of herbs. This study reports herbal remedies as a cause of $\mathrm{MC}$ along with laxatives [9]. It is clear that senna-containing teas or herbals could lead to MC. To date, there is no study investigating $\mathrm{MC}$ and the use of anthranoid-containing herbal remedies without a history of laxative drug use.

We aimed to report our experience with $M C$ and to compare the results with published data with special emphasis on herbal medicine as the primary offending agent.

\section{Material and Methods}

This is a case series study to identify the clinical characteristics and etiology of $\mathrm{MC}$ in affected patients.

Between 2005 and 2006, all consecutive patients who underwent total colonoscopy at the endoscopic unit of 29 Mayis Hospital were considered for evaluation. This is a private hospital in Ankara serving the general population as a secondary-care center. The gastroenterology practice is busy, with the ability to perform most of the diagnostic and therapeutic interventions. Pathological examinations are outsourced to a private out-ofhospital pathology center. The endoscopy center has been working as an open-access center responding to requests from other departments in the hospital. After the index case of MC with consumption of herbal teas containing senna, we decided to search the role of herbal teas, remedies or medicines in the development of MC. We included patients with an endoscopic diagnosis of MC. We had a total of 380 colonoscopies performed during the stated period. We excluded leftsided colonoscopies and sigmoidoscopies, although we did not in fact have any patient diagnosed with MC during these procedures.

The same endoscopist (T.K.) did all the colonoscopies. The examinations reached as far as the terminal ileum in most of the cases. Mucosal changes were noted for the distribution, severity and form of the pigmentation.
Endoscopic diagnostic criteria for MC were dark pigmentation of the mucosa from light brown to black, sometimes with a leopard-skin or reticulated pattern, and dilated colon segments with loss of haustra. As MC usually affects the proximal colonic segments first, special care was given to any discoloration of the proximal colon; even hardly visible brown speckles were biopsied. All of the patients included in this study had the signs of $\mathrm{MC}$ at colonoscopy.

MC diagnoses were confirmed by pathological examination. Paraffin sections obtained from the caecum and ascending colon were stained with haematoxylin and eosin (H\&E). Patients with characteristic pigmentladen macrophages found in the lamina propria are considered to have MC.

After the colonoscopic diagnosis of MC, patients were interviewed by an investigator. Patients were investigated for the long-term use of laxatives, presence of constipation or diarrhea, and other clinical findings. Patients with MC were also asked for the use and length of use of any type of complementary and alternative medicine practices, especially herbal remedies for various reasons. We received information on usage, duration, and type of CAM. Patients were asked for any benefit from CAM. Patients were also asked for knowledge about CAM practices such as side effects and any side-effect experience. Finally, we asked if they had provided information to their physician about using herbal remedies regularly before any decision was made to schedule an examination.

\section{Results}

A total of 380 colonoscopies were performed between 2005 and 2006. Successful colonoscopy was achieved in 346 patients, thereby reflecting a success rate of $90 \%$. Reasons for unsuccessful colonoscopies were inadequate bowel cleansing in 22 patients, the patient's nontolerance and pain in 4 cases, and technical difficulty due to redundant bowel segments in 8 patients.

During this period, 12 patients were endoscopically diagnosed as having MC. MC incidence among patients who underwent colonoscopy was $3.16 \%$. Most of the patients were female; the female-to-male ratio was 10/2. The patient mean age at the time of diagnosis was 53.9 years (min: 46; max: 79). After histolopathologic examination, one patient (Case 5) was found to have amebiasis, although hardly visible brown speckles were observed via endoscopy.

Two patients (18\%) had pancolonic involvement with diffuse discoloration of the colonic mucosa. Our index patient (Case 10) had a heavy brown discoloration and 
Figure 1. Heavy brown discoloration and reticulated pattern in the colonic mucosa.

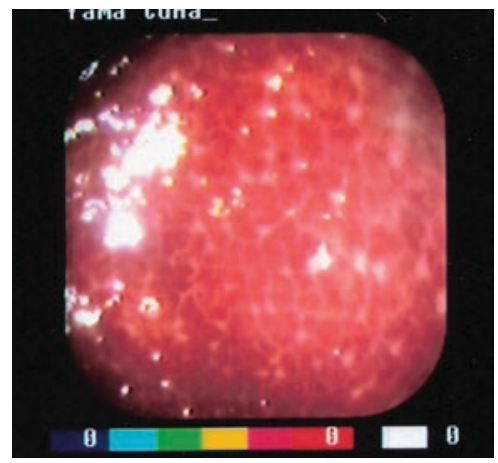

Figure 3. White plaques suggestive of adenoma are easily distinguished against dark colored mucosa.

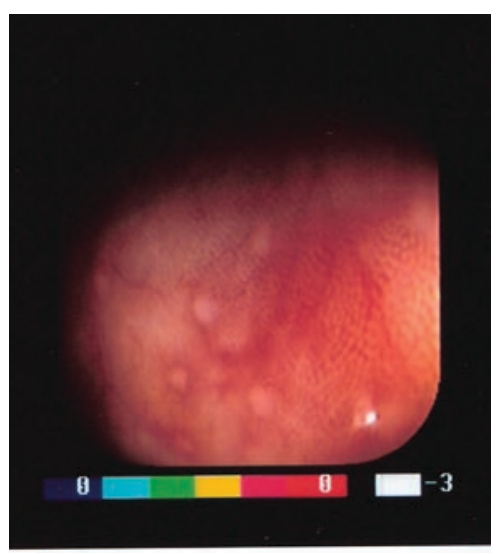

reticulated pattern in the colonic mucosa (Figure 1). The other patient with pancolonic involvement had diffuse light brown discoloration and scattered oval-shaped white areas measuring around $2 \mathrm{~mm}$, suggesting adenoma formation; however, histology analysis showed no adenoma. Both patients had a history of long-term use of senna-containing teas (at least twice
Figure 2. Hardly visible speckles in the colonic mucosa.

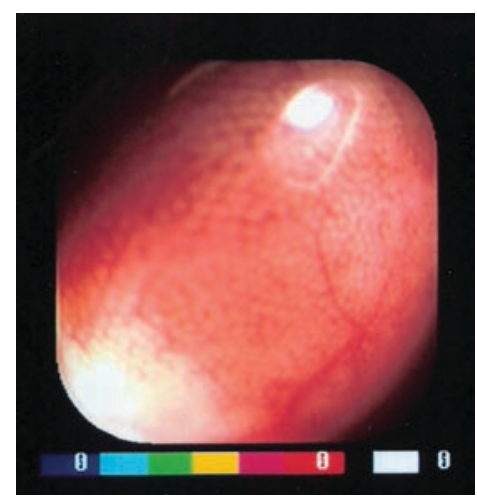

a day). The rest of the patients (82\%) had proximal disease, which was confined to the caecum and ascending colon. Endoscopic features of these patients were less pronounced when we compared them to diffuse involvement. In particular, hardly visible speckles (Figure 2) required special attention for diagnosis. Only 3 patients (27\%) had this type of involvement; however, one patient was overdiagnosed with MC based on an initial assumption that the unhealthy appearance was due to MC, which was later found to be due to amebiasis. Overall, 7 patients (64\%) had a very mild form of MC. White plaques suggestive of adenoma (Figure 3 ) were found endoscopically in 2 patients (18\%), but only one flat adenoma was identified. This observation may be due to sampling error.

Clinical characteristics and found etiologic causes are summarized in Table 1. A total of 11 patients were identified as having $\mathrm{MC}$ and asked about the use of CAM practices. Only one patient $(9 \%)$ had a history of long-term use of anthracene-containing laxatives. One patient $(9 \%)$ denied any use of laxative drugs or herbal teas. The rest of the patients $(n=9,81 \%)$ used Cassia angustifolia teas. The reasons given for herbal

Table 1. Patients' demographical features, colonoscopy indications and any laxative use.

\begin{tabular}{l|lll}
\hline Case & Age & Gender & Colonoscopy indication \\
\hline \hline 1 & 59 & F & Constipation, bloating \\
2 & 55 & F & Constipation \\
3 & 50 & F & Constipation \\
4 & 47 & F & Constipation rectal pain and rectal bleeding \\
5 & 47 & F & Diarrea \\
6 & 55 & M & Change in bowel habits \\
7 & 55 & F & Surveillance \\
8 & 46 & F & Change in bowel habits \\
9 & 48 & F & Change in bowel habits \\
10 & 79 & F & Diarrhea \\
11 & 50 & F & Constipation \\
12 & 56 & M & Constipation \\
\hline
\end{tabular}


Table 2. Type of herbal remedies, duration of use.

\begin{tabular}{l|llllll}
\hline Case & Herbal remedy & Reason & Duration & Still using & Any benefit & Side effects \\
\hline \hline 1 & Senna Tea & Bloating & $2 \mathrm{yrs}$ & $\mathrm{Y}$ & $\mathrm{Y}$ & Diarrhea \\
2 & Senna Tea & To Lose weight & $4 \mathrm{mos}$ & $\mathrm{N}$ & $\mathrm{N}$ & Dyspepsia \\
3 & No & N/A & $\mathrm{N} / \mathrm{A}$ & $\mathrm{N} / \mathrm{A}$ & $\mathrm{N} / \mathrm{A}$ & $\mathrm{N} / \mathrm{A}$ \\
4 & Senna tea & constipation & $1 \mathrm{yr}$ & $\mathrm{Y}$ & $\mathrm{N}$ & $\mathrm{N}$ \\
5 & Senna tea & Bloating & $6 \mathrm{mo}$ & $\mathrm{Y}$ & $\mathrm{N}$ & $\mathrm{N}$ \\
6 & Senna tea & Bloating & $1 \mathrm{yr}$ & $\mathrm{N}$ & $\mathrm{N}$ & $\mathrm{N}$ \\
7 & Senna tea & Habitual & $4 \mathrm{yr}$ & $\mathrm{Y}$ & $\mathrm{N} / \mathrm{A}$ & $\mathrm{N}$ \\
8 & Senna tea & To regulate bowel movements, bloating & $6 \mathrm{mo}$ & $\mathrm{Y}$ & $\mathrm{N}$ & $\mathrm{N}$ \\
9 & Senna tea & Constipation & $2 \mathrm{yr}$ & $\mathrm{Y}$ & $\mathrm{N}$ & $\mathrm{N}$ \\
10 & Senna tea & To regulate bowel movements, bloating, habitual & $10 \mathrm{yrs}$ & $\mathrm{Y}$ & $\mathrm{Y}$ & Diarrhea \\
11 & Senna tea & Constipation & $6 \mathrm{yrs}$ & $\mathrm{Y}$ & $\mathrm{N}$ & $\mathrm{N}$ \\
12 & No & N/A & $\mathrm{N} / \mathrm{A}$ & $\mathrm{N} / \mathrm{A}$ & $\mathrm{N} / \mathrm{A}$ & $\mathrm{N} / \mathrm{A}$ \\
\hline
\end{tabular}

Y: Yes, N: No, N/A: Not applicable

Table 3. Distribution, characteristics and histologic diagnosis of patients.

\begin{tabular}{l|lll}
\hline Case & Distribution & Pattern & Histology \\
\hline \hline 1 & Caecum & Hardly visible speckles & Melanosis coli (MC) \\
2 & Caecum & Mild discoloration & MC \\
3 & Caecum & Hardly visible speckles & MC \\
4 & Caecum & Brown discoloration & MC \\
5 & Caecum, ascending colon & Hardly visible speckles & Amebiasis, no pigment laiden macrophages \\
6 & Caecum & Granulation and discoloration & MC \\
7 & Pancolonic & Diffuse discoloration and white areas & MC but no adenoma found \\
8 & Caecum, ascending colon & Diffuse discoloration & MC \\
9 & Caecum, ascending colon & Brown speckles & MC \\
10 & Pancolonic & Reticulated, heavy Brown discoloration & MC \\
11 & Caecum, ascending colon & Light Brown discoloration, white areas & MC with adenoma \\
12 & Caecum & Light Brown discoloration & MC \\
\hline
\end{tabular}

remedy use were as follows (Table 2): constipation, bloating, losing weight, and habitual usage. Not all of the patients informed their doctor about CAM use during the thorough history taking, but all of them mentioned the ingestion during the interview. None of them had enough knowledge of the side effects.

Duration of usage is between 4 months to 10 years. Only 2 of the patients declared any benefit, and 4 patients were still using the teas. All the patients using herbal remedies relied on information from friends and family.

Endoscopic appearance, distribution and histological diagnosis of patients are outlined in Table 3.

\section{Discussion}

The term melanosis (Greek: melas, black; osis, condition) refers to conditions in which there is an abnormal grayish black or brownish black pigmentation of an organ. The term only implies that the pigment is brown, not specifically melanin. MC is an abnormal brown or sometimes black pigmentation of the colonic mucosa due to long-term anthranoid laxative use. It was first described by Cruveilhier, and Virchow used the term melanosis coli. Early histochemical examinations suggested the pigment deposited is melanin or a melaninlike substance, although in the past microscopists repeatedly reported that no pigment is seen in the mucosa except in submucosal macrophages. It is true that most of the pigment granules do lie in the submucosa. The wall of the colon may appear grossly normal, but microscopic examination may show areas of pigment. Histologically, the epithelial cells are unremarkable. The mucosa and submucosa are usually edematous and contain pigment-laden macrophages and some plasma cells and mast cells [10].

The anthraquinones often occur in plants in the form of glycosides and have colors ranging from yellow to red. 
These compounds are found in rhubarb root, senna leaf, pod, cascara sagrada, frangula, and aloe plants [1,11].

An active derivative, rhein, is formed in the large intestine by bacteria, which causes injury to the cells in the lining of the intestine and leads to apoptosis. The apoptotic cells appear as darkly pigmented bodies that are taken up by macrophages. When sufficient cells have been damaged, the distinctive pigmentation of the bowel wall develops [1,11-13].

MC has no specific symptom on its own; instead, symptoms may be attributed to such accompanying conditions as chronic constipation or diarrhea. We found chronic constipation, changes in bowel habits and diarrhea in 1 patient. This patient had been using the herbal tea for bloating and was aware of neither the side effects nor that this tea was the cause of her chronic diarrhea.

The intensity of the discoloration may differ from barely discernible brown coloration to black. Close examination may reveal small $(2 \mathrm{~mm}$ to $1 \mathrm{~cm})$ raised areas separated by a thin unpigmented reticulum ("toad back" appearance) [10]. In our cohort we have seen discoloration in the colon varying from barely noticed speckles to heavy dark brown. Because of the nature of the changes, it is easy to recall $M C$ in a patient with diffuse heavy discoloration. During colonoscopy, special attention is given to mucosal integrity or irregularity, submucosal vessels, and changes in the colonic lumen. We also examine the colorof the mucosa (such as redness or pallor) for clues to any underlying disease. Due to time constraints, discoloration may be overlooked if it is suggestive of benign causes. Inadequate bowel cleansing may be another factor contributing to not identifying the discoloration of MC.

In humans, and likewise in guinea pigs, macrophages are most abundant in the caecum, decreasing towards the rectum. This explains why the most frequent sites of involvement are the caecum and the appendix, which is the usual distribution pattern of MC, in the proximal as opposed to the distal colon, though it may affect the whole length of the large bowel $[10,14]$.

We had only two patients with the diffuse form affecting the whole length of the colon. Other patients had proximal involvement as expected. Most of them were confined to the caecum and ascending colon. Long-term use of herbal remedies was believed to be a cause in one patient, while another one had been using laxatives for several years. If intake of herbals is stopped, the mucosal color returns to normal after almost a year. Some of the patients were not using herbals during the colonoscopy, though the time of cessation was not requested. For some of the patients with light brown speckles, discontinuation of the offending agent and healing mucosa could be the cause.

Anthraquinone compounds are present in many over-the-counter laxatives, including herbal medicines. In addition, laxatives are frequently used as slimming agents, usually in the form of herbal teas. With a sufficient dose and duration of laxative ingestion, the entire colon may be involved. MC can be developed in as short as 6 weeks. In this cohort, most of the patients were shortterm users (from 3 months to 3 years), whereas the history of laxative use was 10 years in a patient with diffuse involvement, which supports the argument that long-term use of senna-containing herbal teas could lead to development of MC. Laxative drug use was rare in our patient group, but 10 out of 11 patients admitted the use of herbal medicine for various reasons.

A study from Turkey investigated the 13 different preparations of diet-form teas, which could be accessed easily for their herbal components. Senna was the most commonly used plant in the herbal-form tea preparations marketed in Turkey. Some of the preparations also contain aloe, which increases the amount of anthraquinone more than desired [8]. There is no study investigating the incidence of CAM use in Turkish patients with gastrointestinal disorders. Most of the Turkish literature on CAM use is focused on cancer patients.

Cassia angustifolia is also known as "sena maki" among Turkish people, and extracts (liquid or solid) are used. Leaflets of senna contains at least $2.5 \%$ of hydroxyanthracene glycosides the in the form of sennosides $A$ and $B$ [14]. Tea of senna was ingested regularly by 10 patients. The shortest duration of use that was enough for the development of MC was 3 months.

The use of CAM has been on the rise in the past few decades. Almost half of patients with gastrointestinal disorders have tried some form of CAM. CAM use appears to be more common in the functional gastrointestinal disorders. Patients with chronic and refractory gastrointestinal disorders tend to use more common and herbal products as the most frequent type of CAM. Overall, $10 \%$ of herbal therapy is used for digestive symptoms. Indeed, up to $30 \%$ of patients with chronic liver disease and $40 \%$ of patients with irritable bowel syndrome claim to have used some form of herbal medication [5-7].

Usage appears to be most common in patients with inflammatory bowel disease (IBD). Several studies have shown that female gender is most predictive of CAM use [4,15-17]. Patients are more likely to use CAM as an adjunct to conventional medicine than instead of conventional medicine, and only $48 \%$ of patients taking CAM provide this information to their physician. 
Interestingly, doctors may not question patients about CAM usage [7].

$\mathrm{MC}$ has been reported as a consequence of longstanding IBD [7]. The authors concluded that chronic colitis with increased apoptosis could also cause MC without laxative abuse [18]. We did not have a patient with inflammatory bowel disease; one patient had a hardly visible brown discoloration in the caecum. Biopsy showed mild inflammation, but no pigmentladen macrophages were seen. Patients with IBD tend to use CAM therapies more frequently, which may be the reason why patients with IBD and MC should be questioned for the use of CAM.

Some investigators have suggested that increase in apoptosis of colonic mucosa by anthraquinone laxatives increased the risk of colonic cancer. However, recent data, including those from large-scale retrospective, prospective and experimental studies, did not show any increased cancer risk. It is widely accepted that increase in the incidence of adenomas is probably due to easier detection of small adenomas against the black background [2,19-21]. We had only one patient with adenoma and did not see any MC in proximal segments of patients with colon cancer.

Almost all of the patients in this study used herbal teas for gastrointestinal symptoms. Some of them showed serious side effects of laxatives like diarrhea. Patients were neither asked about herbal medicine use nor did they share this information with their primary physician.

Limitations of the study. This study was limited by recruiting patients with findings suggestive of $M C$; therefore, there was no control group to compare with. Although patients included in this study were selected in a prospective manner and asked about CAM and herbal tea usage, we did not investigate the patients with regular CAM use. The findings may therefore have generalization limited to patients consuming herbal teas regularly.

Secondly, neither was other patients undergoing regular colonoscopy asked nor did they specify whether

\section{References}

[1] Muller-Lissner SA Adverse Effects Of Laxatives: Fact And Fiction Pharmacology 1993 Oct;47 Suppl 1:138-45

[2] Nusko G, Schneider B, Schneider I, Wittekind CH, Hahn E GAnthranoid Laxative Use Is NotARisk Factor For Colorectal Neoplasia: Results Of A Prospective Case Control Study Gut 2000;46:651-655

[3] Wald A Is Chronic Use Of Stimulant Laxatives Harmful To The Colon? J Clin Gastroenterol they used CAM therapies for their bowel problems. As we have no data on microscopic MC, we cannot exclude selection bias. The associations between CAM use and bowel disorders are therefore tentative. An additional limitation is the dependence on self-reporting for CAM.

\section{Conclusion}

It is important to emphasize that, particularly in regards to herbal treatments, "natural" or "alternative" is not equivalent to "safe." Side effects, toxicities, and drugherb interactions can occur, and patients should be made aware of this possibility. MC is a harmless mucosal discoloration due to anthraquinone laxatives, which is found to be a common agent consumed in high amounts in herbal tea.

\section{Disclosure Statement}

This research received no specific grant from any funding agency in the public, commercial or non-profit organizations.

\section{Authors' contribution}

TK made the study design, acquisition, analysis, interpretation of data and wrote this article. YB has been involved in revising in critically important content.

\section{Authors' information}

TK works as consultant in gastroenterology in Hacettepe University School of Medicine and has special interest in diagnostic and therapeutic endoscopy

YB is the chief of gastroenterology department at Hacettepe University School of Medicine and has special interest in therapeutic endoscopy and hepatology. $\mathrm{He}$ is a one of the renowned investigator for vascular diseases of the liver and portal system.

2003;36(5):386-389

[4] Tillisch K Complementary And Alternative Medicine For Functional Gastrointestinal Disorders Gut 2006;55:593-596

[5] Comar KM,. Kirby DF Herbal Remedies In Gastroenterology J Clin Gastroenterol 2005;39:457-468

[6] ErnstEComplementaryMedicineIn Gastroenterology: More Than A Fad? J Clin Gastroenterol, Vol 36, No. 


\section{5,P:453}

[7] Kong SC, Hurlstone DP, Pocock CY, Walkington LA, Farguharson NR, Bramble MG, McAlindon ME, Sanders DS. The Incidence Of Self-Prescribed Oral Complementary And Alternative Medicine Use By Patients With Gastrointestinal Diseases J Clin Gastroenterol 2005;39:138-141

[8] Saracoglu A, Ergun B Composition Of Some Herbal Slimming Tea Preparations Marketed In Turkey And Their Possible Adverse Effects Turkiye Klinikleri J Med Sci 2006, 26:355-363

[9] Park WK, Cho KA, Hwang DY Clinical Review Of Melanosis Coli. J Korean Soc Coloproctol. 1999 Aug; 15(3):187-193. Korean

[10] Ghadially FN, Walley VM Melanoses of the Gastrointestinal Tract Histopathology 1994, 25. 197-207

[11] De Witte P, Metabolism And Pharmacokinetics Of Antrhanoids, Pharmacology 1993; 47 (Supplement 1): $86-97$

[12] Van Gorkom BA, De Vries EG, Karrenbeld A, Kleibeuker JH. Review Article:Anthranoid Laxatives And Their Potential Carcinogenic Effects. Aliment Pharmacol Ther 1999;13(4):443-52

[13] Wang X, Zhong YX, Lan M, Zhang ZY, Shi YQ, Lu J, Ding J, Wu KC, Jin JP, Pan BR, Fan DM. Screening And Identification Of Proteins Mediating Senna Induced Gastrointestinal Motility Enhancement In Mouse Colon World J Gastroenterol 2002;8(1):162-167

[14] Folium Sennae, In WHO Monographs On Selected Medicinal Plants Vol. 1 Geneva 1999, Pp: 241 250
[15] Tillisch K Complementary and Alternative Medicine for Gastrointestinal Disorders Clin Med 2007;7(3):224-7

[16] Langmead L, Rampton DS Review article: complementary and alternative therapies for inflammatory bowel disease Aliment Pharmacol Ther 2006 1; 23(3) 341-9

[17] Van Tilburg MA, Palsson OS, Levy RL, Feld AD, Turner MJ, Drossman DA, Whitehead WE. Complementary And Alternative Medicine Use And Cost In Functional Bowel Disorders: A Six Month Prospective Study In A Large HMO BMC Complement Altern Med 2008 Jul 24; 8:46

[18] Pardi DS, Tremaine WJ, Rothenberg HJ, Batts KP. Melanosis Coli In Inflammatory Bowel Disease. J Clin Gastroenterol 1998;26:167-170

[19] Von Gorkom BA, Karrenbeld A, Van Der Sluis T, Zwart N, de Vries EG, Kleibeuker JH. Apoptosis Induction By Sennoside Laxatives In Man; Escape From A Protective Mechanism During Chronic Sennoside Use? J Pathol 2001;194:493-499

[20] Nusko G, Schneider B, Muller G, Kusche J, Hahn EG. Retrospective Study On Laxative Use And Melanosis Coli As Risk Factors For Colorectal Neoplasma. Pharmacology 1993;47 Supl 1:234-241

[21] Nusko G, Schneider B, Ernst H, Wittekind C, Hahn EG. Melanosis Coli - A Harmless Pigmentation Or A Precancerous Condition? Z Gastroenterol 1997;35:313-318 